sources with which we are at present familiar. A third period, which only occurs in the extraordinarily complex system Her X-1, is the 35-day period on which the $\mathrm{X}$-ray radiation in this source seems to be switched on and then switched off, like a regular recurring X-ray flare.

But the new transient source, which was only visible for about ten days, seems according to the Mullard group to have a 6.75 -minute period present in the signal. The problem for the theorists will now be to fit such a period into a binary, mass transfer model. If it is the rotation period of the accreting star it would be extremely, and indeed uniquely, long. If it is the binary orbital period then it would be extremely, and excitingly, short-demanding a very small size for the companion as the source of accreted material. But perhaps, like the 35 day on-off period of Her X-1, it will require a completely different and individual explanation. If other X-ray sources can be taken as an example it will not be long before a plethora of theories are produced trying to account for this totally unexpected new observation by the Mullard group.

Two recently published optical observations of the X-ray source Cen X-3 by Petro and by Osmer, Hiltner and Whelan (Astrophys. J., 195, 705 and $709 ; 1975)$ add a note of caution and sobriety to the world of X-ray sources. The first of these is a series of photoelectric observations of the variations in brightness of the Cen X-3 binary system about its orbit. The second is a study of the spectral type of the optical, mass-losing star. Both conclude that those values for the masses of X-ray binary stans which in the past have been determined on the basis of fitting the spectral type (that is, the appearance of the star's spectrum) and the luminosity (the star's intrinsic

\section{More from Ariel V}

\section{by John Gribbin}

FURTher early results from Ariel V were reported at a press conference on April 17, organised by the Science Research Council to coincide with the publication of the papers describing the source dubbed 'Cen Xmas' (Nature, 254, 577 and $578 ; 1975)$. The UK satellite has in fact already produced two important results apart from the Cen Xmas source. The first is a general comparison with the Uhuru surveys: so far, with $30 \%$ of the first Ariel V survey completed at a sensitivity 3 to 5 times better than the Uhuru 1971-2 surveys, it seems that 16 of the Uhuru sources have disappeared in the past few years. Nineteen new sources have already been found, one (the brightest for a time) being a transient with a lifetime of only a few weeks. This evidence for a changing $X$-ray sky highlights the need for Ariel V (and its successors) to continue sky survey programmes.

The second major news item came from Peter Willmore (now at University of Birmingham) who announced that an investigation of the galactic centre region showed a bright X-ray source in February, and that this source had definitely not been detectable as recently as November 1974. This might not seem too remarkable in view of the frequency with which other sources seem to be popping in and out of view, but of course the galactic centre is a particularly interesting region where events that we can only observe through a dust veil, darkly, seem to be happening at many wavelengths.

The most up to the minute news came from Ken Pounds of the University of Leicester, reporting a second Leicester expeniment which "within the past few days" has "shown positive evidence for spectral lines near $6.6 \mathrm{keV}$ and $2.1 \mathrm{keV}$ " in the supernova remnants Cas A and Tycho's Nova. Claims in a release distributed at the conference that "if confirmed, these will be the first positively identified X-ray lines from a cosmic source" may seem a little exaggerated to those who remember the days when Sco X-1 was the only known non-solar cosmic X-ray source; it depends, perhaps, on the definition of "positively identified", but there has long been quite convincing evidence that iron lines have been detected on occasion in the flux from Sco X-1.

But that does not detract from the importance of these observations, which suggest an abundance of heavy elements in the remnants several times greater than the cosmic norm, nicely in agreement with the generally accepted view that all elements except hydrogen and helium are synthesised in stars and distributed through the galaxies by nova and supernova events.

Where next for Ariel V? Peter Sanford (Mullard Space Science Laboratory) says that the satellite of years, so the story is far from over. should have a useful life of a couple brightness) to theoretical stellar models can be seriously in error. This is extremely important in the present search for black holes in X-ray sources. For it has been claimed that the masses of the compact X-ray sources in several systems must be greater than the theoretical maximum possible mass for a neutron star. But these two papers demonstrate conclusively that one must be extremely careful in determining the orbital parameters of such X-ray binary systems. Simpleminded arguments based on our general understanding of single-star structure may be seriously in error when applied to the pathological X-ray binaries.

Nonetheless, this work on the Cen X-3 system does not dispose of the problem presented by the system Cyg X-1, in which the $\mathrm{X}$-ray sounce is almost certainly more massive than the critical neutron star mass. Like the grin of the Cheshire cat this conclusion does not seem to want to fade away. The evidence for the existence of a black hole in the Cyg X-1 system is still, as yet, neither substantial enough to confound the sceptics, nor weak enough for them to dismiss it. Demonstration using the techniques of these two papers that Cyg X-1 was not a unique system would have helped in resolving this critically important issue. Now we must wait either for the sceptics themselves to fade away, or for further dramatic news from present, and future, X-ray satellites.

\section{Meteors by television}

\section{from David $W$. Hughes}

NEw techniques are always welcome and meteor scientists have acquired one: television. The time-honoured method of observing meteors-standing outside on a clear moonless night and gazing intently at the sky-still provides many useful results. About ten meteors per hour can be seen; the eye can detect meteors down to about the fifth magnitude (produced by particles with masses in excess of $0.1 \mathrm{~g}$ ) in the direct line of vision. The field of view is a cone with an apex angle of around $120^{\circ}$ but sensitivity to the meteor is strongly dependent on its position in the field of view: the possibility of a meteor far from the direct line of vision being seen decreases drastically with decreasing magnitude.

This field of view restriction can be alleviated by using a camera such as the Baker Super-Schmidt meteor camera. This has a low $f$ number (0.8), a circular field of view $55^{\circ}$ in diameter, uses high speed film and can photograph 